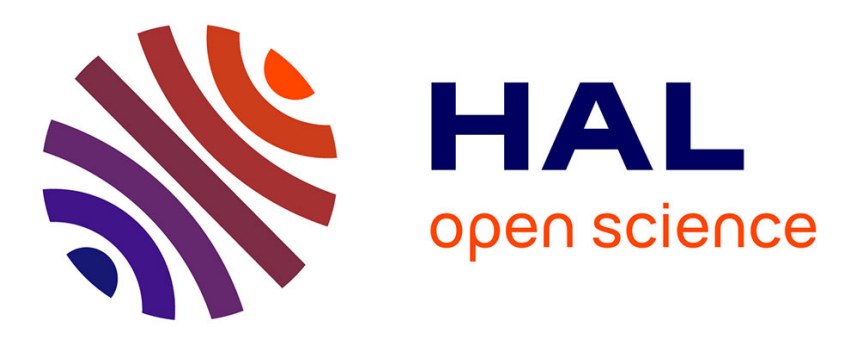

\title{
Source separation and distributed sensing: The key of an efficient monitoring
}

\author{
Jerome I. Mars, Edouard Buchoud, Valeriu Vrabie, A. A. Khan, Sylvain \\ Blairon, Guy d'Urso
}

\section{- To cite this version:}

Jerome I. Mars, Edouard Buchoud, Valeriu Vrabie, A. A. Khan, Sylvain Blairon, et al.. Source separation and distributed sensing: The key of an efficient monitoring. CAMSAP 2013 - 5th IEEE workshop on Computational Advances in Multi-Sensor Adaptive Processing, Dec 2013, Saint Martin, France. pp.264-267. hal-00923555

\section{HAL Id: hal-00923555 \\ https://hal.science/hal-00923555}

Submitted on 3 Jan 2014

HAL is a multi-disciplinary open access archive for the deposit and dissemination of scientific research documents, whether they are published or not. The documents may come from teaching and research institutions in France or abroad, or from public or private research centers.
L'archive ouverte pluridisciplinaire HAL, est destinée au dépôt et à la diffusion de documents scientifiques de niveau recherche, publiés ou non, émanant des établissements d'enseignement et de recherche français ou étrangers, des laboratoires publics ou privés. 


\title{
Source Separation and Distributed Sensing: the Key for an Efficient Monitoring
}

\author{
Jérôme.I. Mars", Edouard Buchoud ${ }^{\# *}$, Valeriu D. Vrabie ${ }^{ \pm}$, Amir Ali Khan ${ }^{\S}$, Sylvain Blairon * Guy D’Urso * \\ \# GIPSA-Lab, Dept Image and Signal, Grenoble Institute of Technology, BP 46, 38402 Saint Martin d'Hères, France \\ 1 jerome.marsegrenoble-inp.fr \\ * EDF Research and Development, Chatou, France \\ ${ }^{ \pm}$CRESTIC, Université de Reims Champagne Ardennes, France \\ ${ }^{ \pm}$SEECS, National University of Sciences and Technology, Islamabad, Pakistan
}

\begin{abstract}
As a complement to classical sensors, Distributed Optical Fiber Sensors now play a prominent role in several engineering fields and act as an antenna array. Depending of the devices used (Raman, Rayleigh, or Brillouin scattering), measurement record depend on temperature, strain, or pressure profile. As the wanted signal is often hidden by noise and other undesired sources, we can express the problem as a source separation problem. In this paper, we show that with the help of recent techniques based on data decomposition and source separation (PCA, ICA NMF techniques) from the virtual antenna, we can accurately identify water leakages from a noisy Raman spectra or a strain profile from Brillouin spectra with a spatial resolution of $1 \mathrm{~cm}$ instead of 1 meter for classical devices.
\end{abstract}

\section{INTRODUCTION}

Distributed Optical Fiber sensors (DOFS) have been employed in diverse domains like: oil and gas industry [1] for parameter monitoring in extreme environments (temperature, pressure, viscosity, flow, etc.[2]); civil engineering for strain measurement and structural health measurement [3], fire detection (underground structures and tunnels [4]), electrical fault detection and metering [5], pollution control through chemical detectors $(\mathrm{H} 2, \mathrm{CO} 2$, explosive detectors), pipeline leakage control, etc [6], [7], [8]. The optical fiber acting as an intrinsic distributed sensor, DOFS offers numerous advantages over the conventional sensors. The possibility of multiplexing a large number of sensors on a single optical fiber and the flexibility of sensor placement are some of the unrivaled advantages. Now, fiber sensing technology can replace the legacy electronic temperature, pressure and strain gauges [9]. Given that this technology has been field-proven, new applications and services are emerging in harsh environment [10]. These new sensing applications are driven by the quest of the smallest possible changes in temperature, pressure, strain (geomechanics parameters) and the capability to monitor large sites and areas as oil reservoirs, wells, dams, industrial structures with the smallest possible spatial resolution. For instance, the durability of civil infrastructures [11], [12], or the monitoring of specific areas as oil reservoir is a crucial issue that can have major economic, social and environmental impacts. In civil engineering, many aging structures may become vulnerable due to internal erosion, climatic conditions and other natural phenomena.
Optimization of maintenance and extension of service life are difficult challenges, real-time diagnosis of the state of wear/damage of a structure representing a key procedure for monitoring. Different techniques (interferometic, polarimetric, Brillouin, Rayleigh and Raman scattering) are in development to create optoelectronic devices able to measure temperature, strain, pressure strain etc.

Using a combination of the measurement of backscattered light and advanced signal processing, an optoelectronic device called interrogator sees the optical fiber as an array of individual sensors. This array allows the detection, discrimination, and location of events over several kilometres. Using signal processing techniques, it is possible to detect and identify a specific signature (leak, strain etc) and eliminate unwanted events. In this paper, we show that advanced signal processing algorithms (subspace decomposition, source separation and non-negative matrix factorization) are necessary to extract the wanted events with high resolution over noisy data. We present two cases, the distributed temperature sensing sytems for water leakage detection and the distributed Brillouin sensing systems for strain estimation.

\section{ACQUISITION PRINCIPLE}

The main important part of the DOFS is the sensing cable composed of several optical fibers, made in silica, wrapped into a protective coating. The interrogator launches a light pulse at a frequency $v_{0}$, into an optical fiber and records the backscattered light. In frequency domain, three distinct phenomena can be highlighted (Fig. 1.)

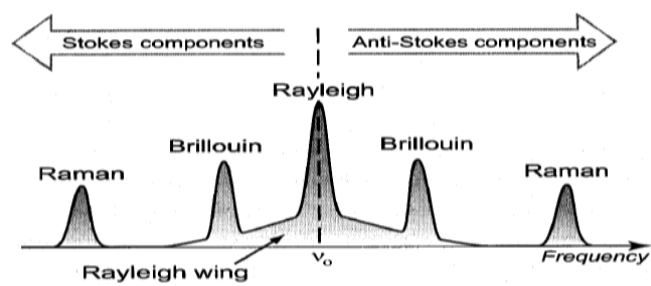

Fig. 1. Backscattering spectrum of a monochromatic wave into an optica fiber exhibiting three scattering phenomena (Rayleigh, Brillouin and Raman) depending on the temperature and/or strain into the silica.

The first one is the Rayleigh scattering: it is due to fluctuations of silica density and composition. The light 
returns with the same frequency $v_{0}$ but parts of the power, called the Rayleigh wing, have been frequency shifted. The second one, the Brillouin scattering due to photon-phonon interaction, is based on the conversion of hydrostatic pressure into distributed mechanical strain acting along a specially coated optical fiber. Measurements of distributed pressure can be thus inferred by converting the applied hydrostatic pressure into distributed mechanical strain. The Brillouin scattering is characterized by a shifted frequency spectrum in the Stokes and Anti-stokes components (see Fig. 1). Those two phenomena are both sensitive to both temperature and strain changes in the optical fiber. The third one, the Raman scattering, is also a shifted frequency spectrum caused by thermal molecular vibration and is only temperature sensitive.

\section{The Distributed TemPerature SENSING Systems CASE}

The Distributed Temperature Sensing Systems (DTS) are fiber optic cables coupled with Raman scattering. Measuring the travel time and knowing the speed of light inside the fiber, the Optical Time Domain Reflectometry (ODTR) principle allows formulating the temperature as a function of distance along the fiber. Moreover, the long-range measurement capability of up to $20 \mathrm{~km}$ with typical spatial resolution of $1 \mathrm{~m}$ and temperature resolution of $0.05^{\circ} \mathrm{C}$, gives a distinct edge acting as an antenna array. For dike and dam monitoring, several sites along hydraulic power stations have been equipped with DTS to check possible water leakages for French Power Company (EDF). The goal is to extract the information pertaining to leakages (both natural and controlled in our case) in the dike of canal as water leakage across the dike generates a thermal anomaly recordable by the fiber optic cable.

\section{A. Data acquisition}

In the presented study, the optical cable is buried at $1 \mathrm{~m}$ in the dike toe. This cable lays $30 \mathrm{~m}$ from the canal toe and cover two kilometers (Fig. 2). This choice made owing to previous studies and suspicion of water leakages in this area [13]. The configuration presents two distinct elevation levels (Zone 1, from $0.1 \mathrm{~km}$ to $1.25 \mathrm{~km}$ and Zone 2, from $1.25 \mathrm{~km}$ to $2.2 \mathrm{~km}$ ) which are differently exposed to the sunlight (i.e. they will exhibit two different thermal behaviors).

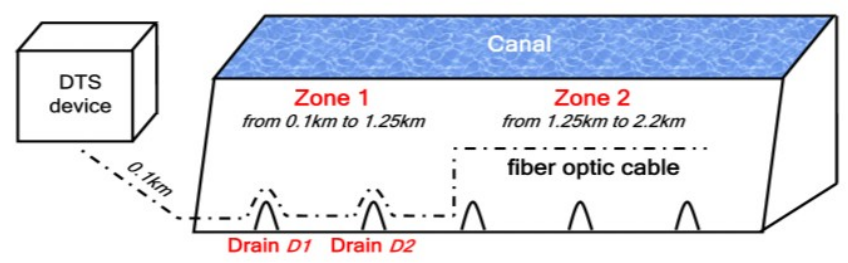

Fig. 2. Site experiment.

In its path, the optical fiber cable longitudinally circumvents two drains labeled hereafter as D1 and D2, provoke a peculiar thermal response and constitute a distinguishable type of anomaly. Along zones 1 and 2, the soil is not homogeneous. During 6 months in 2005, we recorded 12 times a day the temperature along the fiber with $1 \mathrm{~m}$ resolution. In order to validate the acquisition system and leakage detection analysis method, on May $10^{\text {th }}$ and $12^{\text {th }}$, three artificial leakages marked
L1, L2 and L3 (fig. 5), were introduced with different flow rates of 5,1 and $1 \mathrm{lit} / \mathrm{min}$ at the positions: $1.562,1.547$ and $1.569 \mathrm{~km}$, respectively (Red circle on Fig. 3). A hot point (HP) was also introduced on day $10^{\text {th }}$ at $0.674 \mathrm{~km}$. On Fig. 3, spring and summer seasons can be seen (blue showing lowest temperature and red the highest). We also note the behavior of rainfall expressed as line in horizontal striations.

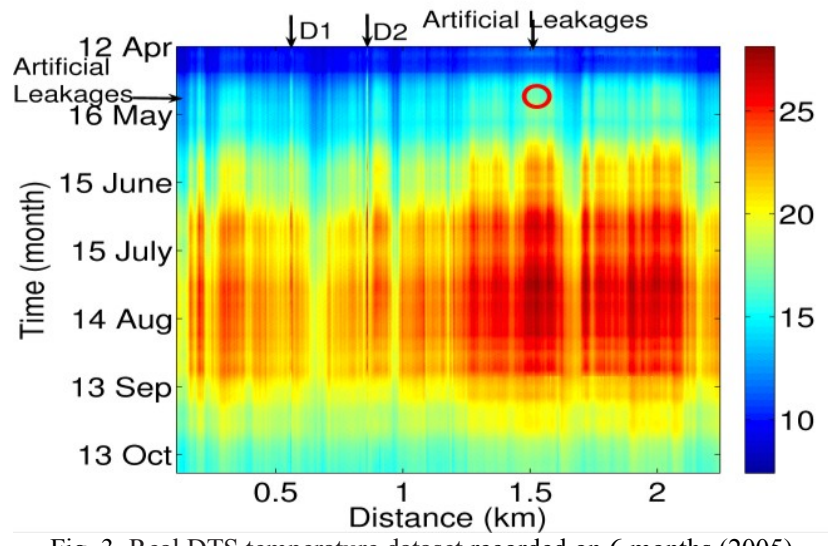

Fig. 3. Real DTS temperature dataset recorded on 6 months (2005).

The identification of the water leakages on the raw data is not straight forward, thus requiring source separation processing to extract information concerning the leakages.

\section{B. Source separation}

To go directly to the wanted signal (leakages), we remove, first, daily (24h) and seasonal effect (month) by a low pass filter and secondly, rainfall periods (hours) by a Kurtosis criteria based filter [14]. After dataset $\mathbf{D}$ can be considered as a linear mixture of independent sources expressed as: $\mathbf{D}^{\mathrm{T}}=$ $\mathbf{M F}^{\mathrm{T}}$, (a source separation problem) with $\mathbf{D} \in \mathrm{R}^{\mathrm{Nx} \times \mathrm{Nt}}$ the acquired data, $\mathbf{M} \in R^{\mathrm{Nt} \times \mathrm{p}}$, the mixing matrix and $\mathbf{F} \in R^{\mathrm{Nx} \times p}$, the $p$ independent sources matrix. The source separation processes used are based on Singular Value Decomp. (SVD) and Independent Component Analysis (ICA) [15-16].

The singular value decomposition of the $\mathrm{D}$ can be expressed

as: $\mathbf{D}=\mathbf{U}_{\mathrm{N}} \boldsymbol{\Lambda}_{\mathrm{N}} \mathbf{V}_{\mathrm{N}}{ }^{\mathrm{T}}=\sum_{j=1}^{m} \lambda_{j} u_{j} v_{j}^{T}+\sum_{j=m+1}^{N} \lambda_{j} u_{j} v_{j}^{T}$

where $\mathrm{N}=\min (\mathrm{Nt}, \mathrm{Nx}), \Lambda_{\mathrm{N}}$ is a diagonal matrix containing the singular values $\lambda_{j} \geq 0$, arranged in a descending order. $\mathbf{U}_{\mathrm{N}}$ and $\mathbf{V}_{\mathrm{N}}$ are orthogonal matrices, containing $\mathrm{N}$ left and right singular vectors $\mathbf{u}_{\mathrm{j}}$ and $\mathbf{v}_{\mathrm{j}}$ respectively. Eq. 1 allows decomposition of data into two subspaces: $\mathbf{D}_{\text {ground }}$ (first $m$ matrices) and $\mathbf{D}_{\text {useful, }}$ containing leakages. The orthogonality condition imposed by SVD is not sufficient for separation of leakages modeled by sparse and non-Gaussian sources. A more realistic approach based on a blind decomposition (ICA), requiring no a priori information about the sources, is applied on the $\mathbf{D}_{\text {useful }}$ subspace. As sources in this context are mutually independent, we estimate them and their weights by maximizing a statistical independence criterion. Details do not fit into the scope of this paper so the interested readers can 
refer to [17]. Taking $\mathbf{D}_{\text {useful, }}$ ICA allows separation of leakage related subspace $\mathbf{D}_{\text {leakage }}$, from the rest of the signal, $\mathbf{D}_{\text {rest }}$, as:

$$
\mathbf{D}_{\text {useful }}=\sum_{j=m+1}^{m+q} \lambda_{j} a_{j} b_{j}^{T} \sum_{j=m+q+1}^{m+P} \lambda_{j} a_{j} b_{j}^{T}
$$

$=\mathbf{D}_{\text {useful }}=\mathbf{D}_{\text {leakage }}+\mathbf{D}_{\text {rest }}$

where $\mathbf{b}_{\mathrm{j}}$ the independent sources obtained from decorrelated ones $\mathbf{v}_{\mathrm{j}}$. The important parameters are: " $m$ ", the number of singular values for building ground subspace, "q", the number of ICA sources to be estimated ( $m$ and $q$ are chosen by eigenvalue decreasing studies). Signal subspace built by the first singular vector is identified as ground response (Fig. 4a) where zone1 and zone2 can be seen. A ground singularity has been also detected (specific soil on site). The first ICA source identifies clearly drains D1 and D2 (Fig. 4b) and the second ICA source gives information on leakage (Fig. 4c).

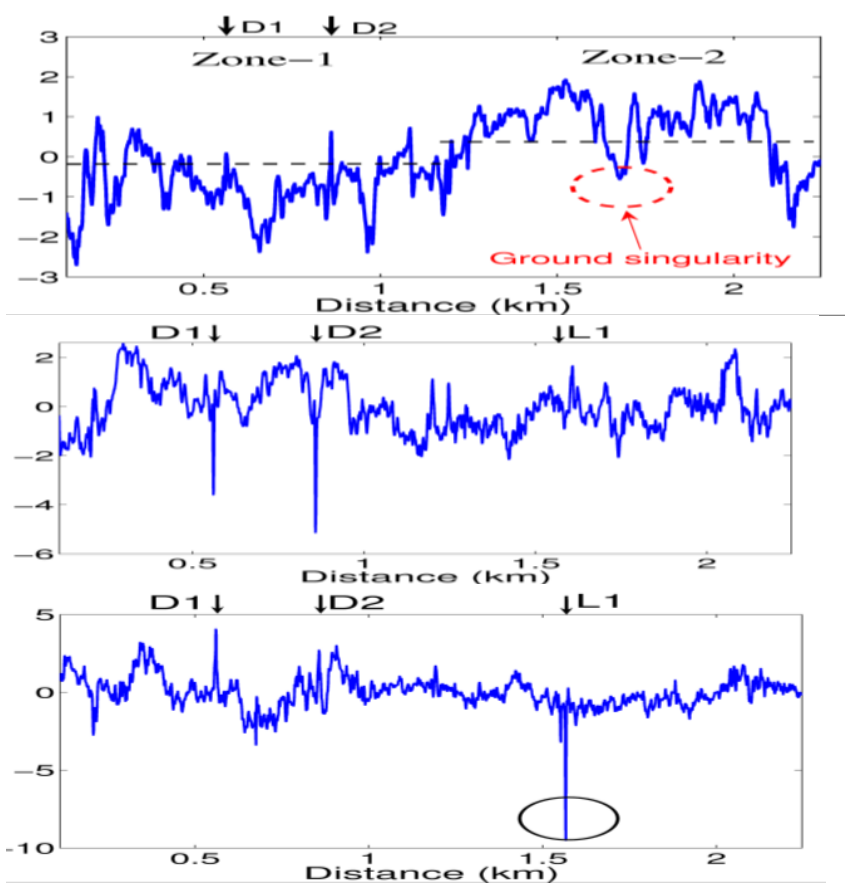

Fig. 4. a) Ground response given by SVD (first singular vector); b) Drain responses given by ICA (first independant vector);

c) Leakage L1 detected by ICA (second independant vector.

In Fig. 5a, final result of the detector on distance axis is proposed. We can identify, each element as drain 1 and 2, leakages 1, 2 and 3 and hot spot. Applying same processing two years latter (2007) on the same site, we find (Fig. 5b), the existing structures (D1, D2) and some real leakages (concentrated around D2) confirmed after visual inspection.
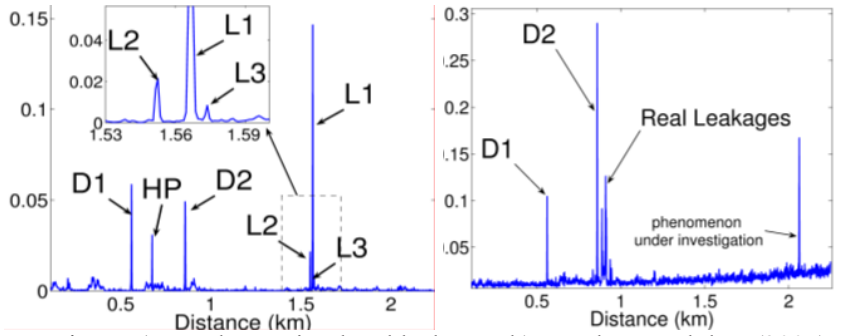

Fig. 5. a) Results on simulated leakages, b) Result on real data (2007)

\section{THE DisTRIBUTED BRILLOUIN SENSORS CASE}

The Distributed Brillouin Sensor (DBS), in comparison with other sensing sensors, has the advantage of combining temperature and strain measurements over few meters to kilometers. This device launches two counter propagative lightwaves into the fiber, one has a fixed frequency, whereas the other is frequency-modulated. An energy transfer, called Brillouin gain, occurs when the two lightwaves encounter each other. The maximum energy transfer occurs when difference between two frequencies equals the Brillouin frequency $v_{B}$ in the optical fiber. This frequency is linearly dependent on the temperature and strain [18]. The spatial resolution $w$ of this device is defined by the wavelength of lightwave pulse. Estimation of strain in the optical fiber is based on the determination of the Brillouin frequency, defined as the abscissa of the maximum of Brillouin spectrum and calculated by fitting Lorentzian or pseudo-Voigt curves [19]. The numerical Brillouin spectrum $G_{z}(v)$, within the spatial resolution, $w$, is modelled as the integral of all the elementary spectra $S_{e}(v)$ centered in the local Brillouin frequencies:

$\widetilde{G}\left(v, z_{i}\right)=\sum_{z_{i}-w / 2}^{z_{i}+w / 2} S_{e}\left(v-v_{B}(x)\right) d x$

If local strains or loads are applied on the fiber at position $\mathrm{z}$, the Brillouin spectrum is distorted [20] and several spectral components $S_{e}(v)$ appear. Their central frequencies and amplitude are related to the strains. Decomposition of a distorted Brillouin spectrum allows determining these spectral components and an estimation of a strain profile at each position z. Several unmixing techniques exist to decompose spectra. Therefore methods like nonnegative matching pursuit [21], Nonnegative Matrix Factorization (NMF) [22] can be used as a Brillouin spectrum is positive.

\section{A. Spectral source separation}

The problem can be expressed as: from three real and positive matrices, $M \cong S A$ where $M \in R_{+}^{N_{f} \times N_{d}}$ the data, $S \in R_{+}^{N_{f} \times N}$ the sources and $A \in R_{+}^{N \times N_{d}}$ their weight and $\mathrm{N}$ the number of sources to estimate, the NMF technique allows to estimate $A$ and $S$ minimizing $F$ [22] as: $F=\|M-S A\|_{2}^{2}$. When strain is uniform, the Brillouin spectrum is an elementary spectrum $S_{e}(\mathrm{v})$. The matrix $S$ is shifted versions of the elementary spectrum with a $\Delta v$ step. The update rules from Lee and Seung [22] are used to minimize F. To be efficient, we regularize the number of possible $\mathrm{N}$ sources through a sparsity criterion [23] defined as:

$$
\operatorname{spa}\left(A_{n}\right)=\frac{\sqrt{N_{d}}-\left(\sum_{x=1}^{N_{d}}\left|a_{n x}\right| / \sqrt{\sum_{x=1}^{N_{d}}\left|a_{n x}^{2}\right|}\right)}{\sqrt{N_{d}}-1}
$$

So the proposed algorithm enables to find a weight vector $\underline{a}$ for each $k^{\text {th }}$ Brillouin spectrum. 


\section{B. Real example}

A controlled experiment has been carried out to validate our methodology. The experimental bench enables to pull the cable constantly between two fixed points $(10.5 \mathrm{~m}$ and $14 \mathrm{~m})$. with two measurements: 1) cable relaxed; 2) cable pulled. Output of device estimate the strain profile with a fixed rate sample distance $\Delta z=40 \mathrm{~cm}$ (Fig 6.b purple curve). By applying the proposed NMF source separation, we obtain the sparsity criteria curve along the distance (fig 6a) based on [24]. This curve highlights 2 points at $z_{1}=10.4 \mathrm{~m}$ and $z_{2}=14,2 \mathrm{~m}$ (distances with the highest strain). As we can choose our rate sample in the NMF procedure $(1 \mathrm{~cm}$ instead of $40 \mathrm{~cm})$, we obtain a better resolution strain profile (fig 6b. black curve). The relative error between a reference strain and the source separation procedure is given in fig. $6 \mathrm{c}(5 \%)$.
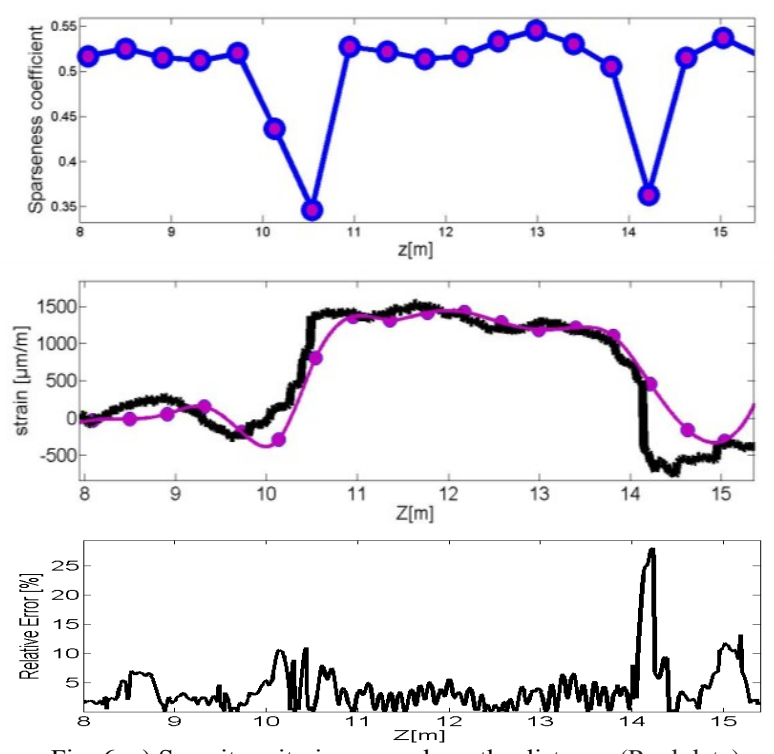

Fig. 6. a) Sparsity criteria curve along the distance (Real data) b) Strain profile from the device (purple), from the NMF processing (black) c) Relative error between reference and the output of NMF

\section{CONCLUSION}

The optical fiber sensing acts as an array and allows detection, location and discrimination of events depending on temperature and/or strain measurement over distance. Source separation processing is proposed to highlight the wanted signature. We prove by SVD-ICA and NMF algorithm on Raman based (temperature) recordings and Brillouin spectrum (strain) that leakage detection, strain profile estimation strain profile and resolution of the result are highly enhanced.

\section{ACKNOWLEDGEMENTS}

We thank the five reviewers for their constructive remarks.

\section{REFERENCES}

[1] Kersey, A. D., "Optical fiber sensors for downwell monitoring applications in the oil and gas industry." Proc. $13^{\text {th }}$ Intl. Conf. Optical Fiber Sensors. 326-331, 1999.

[2] Hartog, A. H.,. Distributed fiber-optic temperature sensors: principles and applications. Optical Fiber Sensor Technology, K. T. Grattan and B. T. Meggitt, eds., Kluwer, 2000
[3] Grosswig, S., Graupner, A., and Hurtig, E., "Distributed fiber optical temperature sensing technique - a variable tool for monitoring tasks." Proc. $8^{\text {th }}$ Intl. Symposium on Temperature and Thermal Measurements in Industry and Science. 9-17, 2001

[4] Hartog, A. H., "Progress in distributed fiber-optic temperature sensing." Proc. SPIE, Fiber Optic Sensor Technology and Applications, M. A. Marcus and B. Culshaw, eds., Vol. 4578 of 0277 786X/02. 43-5, 2002.

[5] Henderson, P. J., Fischer, N. E., and Jackson, D. A., "Current metering using fiber grating based interrogation of a conventional current transformer." Proc. 12 $2^{\text {th }}$ Intl. Conf. Optical Fiber Sensors, Williamsburg, VA. 186-189, 1997.

[6] Fry, J. J., "Internal erosion and surveillance." $19^{\text {th }}$ Annual Meeting of the International Commission on Large Dams Congress, Florence. 1997.

[7] Wade, S. A., Grattan, K. T., and McKinley, B., "Incorporation of fiberoptic sensors in concrete specimens: Testing and evaluation." IEEE Sensors Journal, Vol. 4(1), 127-134, 2004.

[8] Nikles M., "Long-distance fiber optic sensing solutions for pipeline leakage, intrusion and ground movement detection", Fiber Optic Sensors and Applications VI, Proceedings, Vol. 7316, 2009.

[9] Yin S. and Yu F. T. S., "Distributed fiber optic sensors", Optical Engineering, New-York Marcel, 2002.

[10] Lanticq V., Gabet R., Taillade F. and Delepine-Lesoille S.,'Distributed optical fibre sensors for Structural Health Monitoring: Upcoming challenges", Optical Fiber, New developments, 2009.

[11] Hénault J. M., Quiertant M., Delepine-Lesoille S., Salin J., Moreau G., Taillade F., and Benzarti K., "Quantitative strain measurement and crack detection in RC structures using a truly distributed fiber optic sensing system", Construction and Building Materials, Vol. 37, pp. 916-923, 2012.

[12] Nikles M., Burke B., Briffod F. and Lyons G., "Greatly extended distance pipeline monitoring using fiber optics", Proceedings of OMAE05, 24 ${ }^{\text {th }}$ International Conference on Offshore Mechanics and Artics Engineering, June, 12-17, Halkidiki, Greece, 2005.

[13] Fry, J. J., "Détection de fuite sur les digues par acquisition de profils de temperature le long d'une fibre optique." Securite des digues fluviales et de navigation, Actes de colloque du CFGB, Orléans, France. (in French), 2004.

[14] Khan, A. A., Vrabie, V., Mars, J. I., Girard, A., and d'Urso, G., "A least square approach for bidimensional source separation using higher order statistics criteria." EU SIPCO, Lausanne, Switzerland, 2008.

[15] Klema, V. C. and Laub, A. J., "The singular value decomposition: its computation and some applications." IEEE. Trans on Auto. Control, 25(2), 164-176, 1980

[16] Comon, P. and Jutten, C., Handbook of Blind Source Separation. Elsevier, 2010.

[17] Hyvarinen, A., Karhunen, J., and Oja, E., Independent Component Analysis. Wiley New York, 2001.

[18] Bao X., Dhliwayo J., Heron N., Webb D. J. and Jackson D. A., "Experimental and theorical studies on a distributed temperature sensor based on Brillouin scattering," Journal of Lightwave Technology, 13(7), 1995.

[19] Bao X., Brown A., DeMerchant M. and Smith J., "Characterization of the Brillouin-loss spectrum of single-mode fibers by use of very short (<10ns) pulses", Optics Letters, Vol. 23,. 8, 1999.

[20] Ravet F., Bao X., Ozbakkaloglu T. and Satacioglu M., "Signature of structure failure using asymmetric and broadening factors of Brillouin spectrum", IEEE photonics technology letters, Vol. 18(2), 2006.

[21] Mallat S. G. and Zhang Z., "Matching pursuits with time-frequency dictionaries", IEEE Transactions on Signal Processing, Vol.41(12), pp 3397-3415, 1993

[22] Lee D. D and Seung H. S., "Algorithm for non-negative matrix factorization", Advances in neural information processing systems, vol.13, pp. 556-562, 2001

[23] Hoyer P.O., "Non-negative matrix factorization with sparseness constraints", The Journal of Machine Learning Research, Vol.5, pp. 1457-1469, 2004.

[24] Buchoud E., Blairon S., D’Urso G., Henault J. M., Girard A., Mars J. I. and Vrabie V., "Detection of ground movement using the shape of Brillouin spectrum", Near Surface Geoscience 2012, Paris, 2912. 